\title{
Clinical Outcome of Cytoreductive Surgery Prior to Bevacizumab for Patients with Recurrent Glioblastoma: A Single-center Retrospective Analysis
}

\author{
Shigeru YAMAGUCHI, ${ }^{1}$ Hiroaki MoTEGI, ${ }^{1}$ Yukitomo ISHI, ${ }^{1}$ Michinari OKAMOTO, ${ }^{1}$ \\ Ryosuke SAWAYA, ${ }^{1}$ Hiroyuki KOBAYASHI, ${ }^{2}$ Shunsuke TERASAKA, ${ }^{2}$ \\ and Kiyohiro HOUKIN ${ }^{1}$ \\ ${ }^{1}$ Department of Neurosurgery, Faculty of Medicine, Hokkaido University, \\ Sapporo, Hokkaido, Japan \\ ${ }^{2}$ Kashiwaba Neurosurgical Hospital, Sapporo, Hokkaido, Japan
}

\begin{abstract}
Bevacizumab (BEV) is a key anti-angiogenic agent used in the treatment for recurrent glioblastoma multiforme (GBM). The aim of this study was to investigate whether cytoreductive surgery prior to treatment with BEV contributes to prolongation of survival for patients with recurrent GBM. We retrospectively analyzed the treatment outcomes of 124 patients with recurrent GBM who were initially treated with the Stupp protocol between 2006 and 2019. Given that BEV has only been available in Japan since 2013, we grouped the patients into two groups according to the time of first recurrence: the pre-BEV group $(\mathrm{N}=51)$ included patients who had recurrence before BEV approval, and the BEV group $(\mathrm{N}=73)$ included patients with recurrence after BEV approval. The overall survival after first recurrence (OS-R) was analyzed according to the treatment strategy. Among 124 patients, 27 patients $(19.4 \%)$ received cytoreductive surgery. There were nine cases in the pre-BEV group and 18 cases in the BEV group. Although the mean extent of resection for both groups was almost equal, OS-R was significantly different. The median OS-R was $8.1 \mathrm{~m}$ in the pre-BEV group and $16.3 \mathrm{~m}$ in the BEV group $(P=0.007)$. Multivariate analysis revealed that the unavailability of $\mathrm{BEV}$ postoperatively $(P=0.03)$ and decreasing performance status by surgery $(P=0.01)$ were significant poor prognostic factors for survival after surgery. With the advent of BEV, cytoreductive surgery might provide superior survival benefit at the time of GBM recurrence, especially in cases where surgery can be performed without deteriorating the patient's condition.
\end{abstract}

Keywords: bevacizumab, cytoreductive surgery, glioblastoma multiforme, recurrence

\section{Introduction}

Although several promising drugs have come to the forefront recently, glioblastoma multiforme (GBM) is still a dismal disease because the majority of cases eventually experience tumor recurrence. Since there is no standard therapeutic regimen, treatment options for patients with recurrent GBM are usually individualized and diverse. A previous randomized

Received August 26, 2020; Accepted November 20, 2020

Copyright $\subseteq 2021$ by The Japan Neurosurgical Society This work is licensed under a Creative Commons AttributionNonCommercial-NoDerivatives International License. clinical trial found that the median overall survival after recurrence (OS-R) was only 6.2 months. ${ }^{1)}$

The Food and Drug Association in the United States approved bevacizumab (BEV), a humanized monoclonal antibody that inhibits vascular endothelial growth factor, in 2009 for recurrent GBM. BEV was approved in 2013 for malignant glioma, including newly diagnosed glioblastoma, in Japan. $\mathrm{BEV}$ is frequently given to patients with recurrent GBM despite its limited efficacy for prolongation of OS in these patients. ${ }^{2-5)}$ Although patients sometimes experience rapid regression of recurrent lesions after short courses of BEV, median OS after recurrence was still 9.2 months with BEV monotherapy. ${ }^{5)}$

One of the treatment options for recurrent GBM is repeated surgery; however, the efficacy of surgery 
remains controversial. Several retrospective analyses showed that surgical resection had a survival benefit, ${ }^{6-8)}$ whereas other retrospective analyses demonstrated that resection of recurrent lesions was not significantly associated with increased postprogression survival. ${ }^{9,10)}$ In particular, the role of the cytoreduction of recurrent glioma prior to treatment with BEV remains unclear.

In our institution, we always consider repeated surgery when a patient with GBM suffers from recurrent disease during or after maintenance temozolomide (TMZ) treatment. Therefore, since BEV has been approved in Japan, we have used BEV to treat every patient with recurrent GBM who has undergone maximum possible tumor resection. In this study, we hypothesized that for selected cases of recurrent GBM, cytoreductive surgery before treatment with BEV may comprise a more effective salvage treatment and improve OS. The aim of this study was to evaluate whether cytoreductive surgery for recurrent lesions prolongs survival in the BEV era.

\section{Materials and Methods}

\section{Patient selection}

The local Institutional Review Board at Hokkaido University Hospital approved this study. We retrospectively reviewed the medical records of adult patients with GBM treated in our institution by conventional radiotherapy concomitant with TMZ according to the Stupp protocol ${ }^{11)}$ between 2006 and 2019, with June 2013 representing when BEV was first used to treat GBM in Japan. We identified 212 adult patients with GBM during this period.

\section{Basic treatment strategy for GBM in our institution}

Patients diagnosed with GBM during primary surgery underwent $60 \mathrm{~Gy} / 30 \mathrm{Fr}$ localized radiotherapy concomitant with TMZ according to the Stupp protocol. From June 2013, patients whose tumors continuously progressed during radiotherapy received add-on BEV as intensive consolidation therapy. Otherwise, patients underwent adjuvant TMZ chemotherapy $\left(150-200 \mathrm{mg} / \mathrm{m}^{2}, 5\right.$ days, every 4 weeks) for up to 12-24 courses. Patients also underwent contrast-enhanced MRI every 3 months during and after adjuvant TMZ chemotherapy.

Tumor recurrence was revealed by MRI or clinical deterioration. In cases where it was difficult to distinguish tumor recurrence from radiation necrosis, ${ }^{11} \mathrm{C}$-methionine (MET) positron emission tomography was performed. ${ }^{12)}$

Once a recurrent tumor was observed, we considered first and foremost the resection of recurrent lesions. If the majority of the recurrent lesions were resectable without critical additional neurological deficits and the patient condition was tolerable for surgery, we proposed cytoreductive surgery in each case. BCNU wafers (Gliadel), available in Japan since January 2013, were occasionally implanted after tumor resection at the discretion of the surgeons. After cytoreductive surgery for recurrent tumors, patients resumed or continued adjuvant TMZ until the second recurrence. Since June 2013, patients who underwent gross total resection of recurrent tumors received TMZ continuously at the second recurrence. Then BEV was added to intensify consolidation therapy. Patients who did not undergo gross total resection of recurrent tumors received BEV with or without TMZ.

If the cytoreductive resection of a recurrent lesion would incur additional intolerable neurological deficits, or patients did not accept our proposal for repeated surgery, the patients received second-line chemotherapy. Repeated irradiation was not applied in our institution. If the patient or patient family could not accept further anti-tumor treatments, patients received best supportive care (BSC).

\section{Inclusion criteria}

In this study, we focused on the prognosis after first recurrence in patients with primary GBMs who were treated with the Stupp regimen. Therefore, we excluded patients with GBM from this analysis as follows: (1) patients that did not receive radiotherapy $(\mathrm{N}=24)$; (2) patients that did not receive TMZ $(\mathrm{N}=14)$; and (3) patients that received add-on BEV chemotherapy during or just after radiotherapy because of continuous progression $(\mathrm{N}=17)$. Eventually, we analyzed 157 patients with primary GBM in this study.

\section{Assessment}

According to treatment at the first recurrence, we classified patients into three groups: (1) the cytoreductive surgery group, who received maximum safe resection of recurrent tumor with or without chemotherapy, (2) the second-line chemotherapy group, who received chemotherapy without cytoreductive surgery for recurrent tumors, and (3) the BSC group, who did not receive any anti-tumor treatment after recurrence. The prognosis was assessed by OS after the first recurrence (OS-R). Duration of OS-R was defined as the interval between the date of the MRI study on which the first recurrence was detected and the date of death or last follow-up.

Tumor volumes and the extent of resections were calculated by the planimetry method using $5-\mathrm{mm}$ slice axial T1WI with contrast enhancement, as 


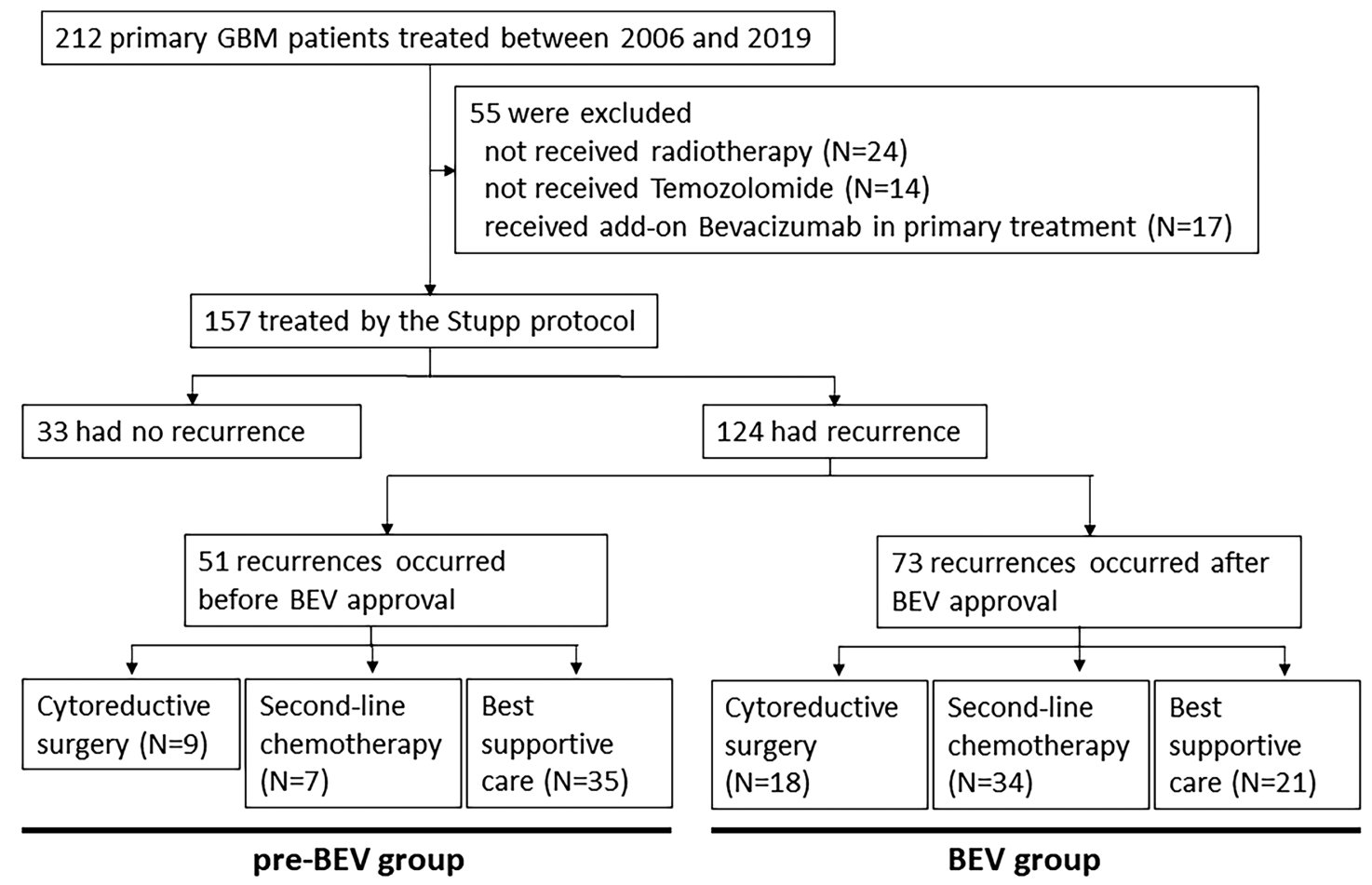

Fig. 1 Inclusion chart and treatment flow of this study according to BEV availability. BEV: bevacizumab.

described previously. ${ }^{13)}$ The extent of resection was defined as the percentage of the resected tumor volume compared to the preoperative tumor volume. In the cytoreductive surgery group, each patient's performance status was assessed by the Karnofsky Performance status (KPS) before and 1 month after the operation. The presence of an isocitrate dehydrogenase (IDH) mutation was determined with direct sequencing for IDH-1 codon 132 and IDH-2 codon $172 . \mathrm{O}^{6}$-methylguanine DNA methyltransferase (MGMT) status was not evaluated in this study.

\section{Statistical analysis}

All statistical analyses were carried out using $\mathrm{R}$ statistical software version 3.4.2. The distribution of categorized data was compared with Pearson's chi-squared test. The means of continuous variables were compared using Welch's $t$-test, and the medians of continuous variables were compared using the Wilcoxon rank-sum test. The Kaplan-Meier method was used to estimate survival curves, and the log-rank test was used for the comparison. To analyze clinical factors for the risk of survival after cytoreductive surgery, Cox proportional hazards regression models were applied. A hazard ratio and 95\% confidence intervals were calculated using multivariate Cox models. In multivariate analysis, clinical factors with a $P$ value $<0.20$ in univariate analysis were selected. Graphic designs were created with
PRISM ver. 8.0. All statistical significance was defined as $P$ value $<0.05$.

\section{Results}

\section{Patient demographics}

This study included 157 primary patients with GBM (90 males and 67 females). The median age of onset was 64 years (range, 25-85 years). Regarding the extent of resection at primary surgery, $18(11.5 \%)$ patients underwent only biopsy, $27(17.2 \%)$ patients underwent partial tumor resection with less than $90 \%$ resection, $43(27.4 \%)$ patients underwent subtotal resection (90-98\% resection), and 69 (43.9\%) patients underwent gross total resection (more than 98\% resection) before treatment with the Stupp protocol. Of these 157 patients, 124 (79.0\%) experienced tumor recurrence and 98 patients $(62.4 \%)$ died at the time of this analysis. Median OS was 20.4 months and the 2-year OS was 39.3\% (Supplement Fig. 1, available online).

\section{Therapeutic approach for the first recurrent GBM}

Among the 124 patients with recurrent disease, 51 recurred before March 2013 (the pre-BEV group). They were unable to receive $\mathrm{BEV}$ in combination with second-line chemotherapy after recurrence; however, three of 51 cases eventually received BEV after its approval in Japan. The remaining 73 patients 
Table 1 Patient Characteristics in 124 recurrent GBMs according to timing of recurrence

\begin{tabular}{lccc}
\hline & $\begin{array}{c}\text { Recurrence before BEV } \\
\text { approval (pre-BEV group) }\end{array}$ & $\begin{array}{c}\text { Recurrence after BEV } \\
\text { approval (BEV group) }\end{array}$ & $P$ value \\
\hline Patient No. & 51 & 73 & 0.01 \\
Age (median) & $62 \mathrm{y}$ & $66 \mathrm{y}$ & $0.22^{\mathrm{a}}$ \\
$\begin{array}{l}\text { Duration from primary surgery } \\
\text { to recurrence (median) }\end{array}$ & 8.2 months & 9.6 months & \\
Treatment after first recurrence & & 18 cases $(24.7 \%)$ & \\
Cytoreductive surgery & 9 cases $(17.6 \%)$ & 34 cases $(46.6 \%)$ & \\
Second-line chemotherapy & 7 cases $(13.7 \%)$ & 21 cases $(28.8 \%)$ & $0.032^{\mathrm{a}}$ \\
Best supportive care & 35 cases $(68.6 \%)$ & 8.1 months \\
Median OS-R & 6.9 months & & \\
\hline
\end{tabular}

${ }^{\text {a }} P$ values were calculated by Log-rank test

BEV: bevacizumab, GBM: glioblastoma multiforme, OS-R: overall survival after recurrence.
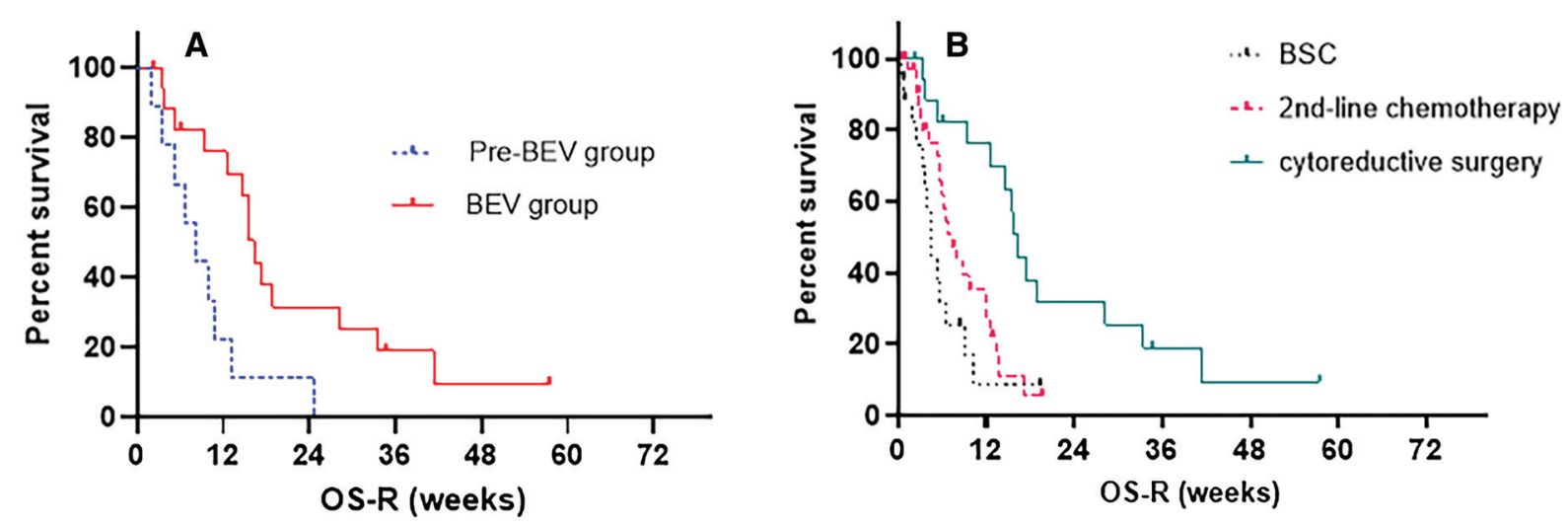

Fig. 2 (A) OS-R in patients who underwent cytoreductive surgery at recurrence are shown according to the timing of recurrence before BEV approval (pre-BEV) or after BEV approval (BEV). Median OS-R of the BEV group (16.3 months) was significantly better than that of the pre-BEV group (8.1 months; $P=0.007)$. (B) OS-R in the BEV group according to the treatment strategy at recurrence. The median OS-R of cytoreductive surgery, second-line chemotherapy without cytoreductive surgery, and best supportive care was 16.3 months, 7.4 months, and 4.6 months, respectively. BEV: bevacizumab, OS-R: overall survival after recurrence.

had tumor recurrence after May 2013, which is when BEV was available for clinical use (the BEV group). Table 1 shows the patient demographics at the time of recurrence. The median duration between primary surgery and recurrence was 8.2 months in the pre-BEV group and 9.6 months in the BEV group. Although the duration of the BEV group was longer than that of the pre-BEV group, this difference was not statistically significant $(P=0.22)$. The therapeutic approach for first tumor recurrence was cytoreductive surgery, second-line chemotherapy, and BSC for 9 (17.6\%), 7 (13.7\%), and 35 patients $(68.6 \%)$ in the pre-BEV group, respectively, and for $18(24.7 \%), 34(46.6 \%)$, and 21 patients $(28.8 \%)$ in the BEV era, respectively. Second-line chemotherapy included add-on interferon- $\beta(\mathrm{N}=3)$, repeat course of TMZ ( $=2)$, BEV after available $(\mathrm{N}=2)$, in the pre-BEV group, while all 34 patients received BEV in combination with second-line chemotherapy in the BEV group. The inclusion chart of this study is shown in Fig 1.

Median OS-R was 6.9 months in the pre-BEV group and 8.1 months in the BEV group. OS-R in the BEV group was significantly longer than in the pre-BEV group regardless of the therapeutic approach ( $P=0.032$; Supplement Fig. 2, available online).

\section{Impact of cytoreductive surgery for first recurrent GBM on OS-R before and after BEV approval}

As described above, cytoreductive surgery for first recurrent GBM was performed in 27 patients, that is, 9 patients from the pre-BEV group and 18 from 
Table 2 Characteristics in 27 patients with recurrent GBM who underwent cytoreductive surgery

\begin{tabular}{|c|c|c|c|}
\hline & Pre-BEV group & BEV group & $P$ value \\
\hline Patient No & 9 & 18 & \\
\hline Age (median) & $67 \mathrm{y}$ & $61 \mathrm{y}$ & 0.33 \\
\hline Preoperative KPS (median) & $80 \%$ & $80 \%$ & 0.37 \\
\hline Laterality (left/right) & 6 cases $/ 3$ cases & 6 cases $/ 12$ cases & 0.10 \\
\hline Tumor location & & & 0.54 \\
\hline Frontal & 1 case & 3 cases & \\
\hline Temporal & 3 cases & 7 cases & \\
\hline Parietal & 4 cases & 8 cases & \\
\hline Occipital & 1 case & 0 case & \\
\hline $\begin{array}{l}\text { Duration from primary surgery to } \\
\text { recurrence (median) }\end{array}$ & 16.9 months & 22.0 months & $0.11^{\mathrm{a}}$ \\
\hline Recurrent tumor volume (mean) & $8.93 \mathrm{~mL}$ & $16.3 \mathrm{~mL}$ & 0.25 \\
\hline Extent of resection (mean) & $92.9 \%$ & $93.3 \%$ & 0.96 \\
\hline BCNU wafer implantation & 1 case $(11 \%)$ & 12 cases $(67 \%)$ & \\
\hline Postoperative KPS (median) & $70 \%$ & $80 \%$ & 0.39 \\
\hline KPS change after surgery (stable/decrease) & 5 cases $/ 4$ cases & 10 cases/8 cases & 1.00 \\
\hline Postoperative Bevacizumab & - & 8 cases $(53 \%)$ & \\
\hline IDH mutation status (mut/wild) & 1 case/8 cases & 1 case/17 cases & 0.51 \\
\hline
\end{tabular}

${ }^{a} P$ values were calculated by Log-rank test

BEV: bevacizumab, GBM: glioblastoma multiforme, IDH: isocitrate dehydrogenase, KPS: Karnofsky

Performance Status.

the BEV group. Table 2 shows the characteristics of the two groups. There were no significant differences between the two groups in age of recurrence, preoperative KPS, laterality, and location of the tumor, duration between primary surgery and recurrence (pre-BEV group: 16.9 months, BEV group: 22.0 months), recurrent tumor volumes, and IDH mutation status.

Almost all patients achieved subtotal resection or gross total resection of recurrent tumors, and the mean extent of cytoreductive surgery was $92.9 \%$ in the pre-BEV group and $93.3 \%$ in the BEV group. In addition, there were no differences in the median KPS score postoperatively between the two groups. Four of nine cases $(44 \%)$ in the pre-BEV group and 8 of 18 cases (44\%) had a declined KPS score one month after the operation. Postoperatively, eight patients $(44 \%)$ were given BEV after recurrent surgery in the BEV group because residual lesions were observed with postoperative MRI.

Eventually, only one patient was given BEV at the second recurrence in the pre-BEV era group after BEV approval. In the BEV group, 15 of 18 cases were treated with $\mathrm{BEV}$; seven patients were given $\mathrm{BEV}$ at the second recurrence. Two patients without BEV application did not have a second recurrence at the time of this analysis. One patient could not receive BEV because of decreasing clinical status caused by surgery. Median OS-R in the pre-BEV group was 8.1 months, while that in the BEV group was 16.3 months (Fig. 2A). The OS-R of the BEV group was significantly longer than that of the pre-BEV group $(P=0.007)$.

In addition, we investigated whether other clinical factors influenced survival after cytoreductive surgery. Table 3 shows the univariate and multivariate analyses of candidate clinical factors. In addition to postoperative BEV availability $(P=0.03)$, the most significant poor prognostic factor affecting OS-R was a decreased KPS score after cytoreductive surgery $(P=0.01)$.

\section{Impact of cytoreductive surgery in the BEV group}

As a second set of analyses, we evaluated whether cytoreductive surgery before treatment with BEV contributes to prolongation of the patient prognosis in the BEV era. There were 73 patients with recurrent GBM in the BEV group, as described above. The median OS-R of patients who received cytoreductive surgery $(\mathrm{N}=18)$, BEV combined with second-line chemotherapy $(\mathrm{N}=34)$, and BSC $(\mathrm{N}=21)$ was 16.3 months, 7.4 months, and 4.6 months, 
Table 3 Univariate and multivariate analyses of clinical factors for survival after cytoreductive surgery by the proportional hazard model

\begin{tabular}{|c|c|c|c|c|c|c|c|}
\hline & & \multicolumn{3}{|c|}{ Univariate analysis } & \multicolumn{3}{|c|}{ Multivariate analysis } \\
\hline & & Hazard ratio & $95 \% \mathrm{CI}$ & $P$ values & Hazard ratio & $95 \%$ CI & $P$ values \\
\hline Age at recurrence & (continuous variable) & 1.001 & $0.97-1.03$ & 0.96 & - & - & - \\
\hline $\begin{array}{l}\text { Interval between } \\
\text { surgeries }\end{array}$ & (continuous variable) & 0.993 & $0.97-1.01$ & 0.47 & - & - & - \\
\hline \multirow[t]{2}{*}{ Preoperative KPS } & $<80 \%$ & 1 & & & 1 & & \\
\hline & $80-100 \%$ & 0.43 & $0.14-1.35$ & 0.15 & 1.20 & $0.31-4.54$ & 0.78 \\
\hline \multirow{2}{*}{$\begin{array}{l}\text { KPS change after } \\
\text { surgery }\end{array}$} & decrease & 1 & & & 1 & & \\
\hline & stable & 0.24 & $0.09-0.66$ & 0.005 & 0.22 & $0.07-0.71$ & 0.01 * \\
\hline $\begin{array}{l}\text { Extent of Resection } \\
(\%)\end{array}$ & (continuous variable) & 0.98 & $0.95-1.02$ & 0.37 & - & - & - \\
\hline \multirow{2}{*}{$\begin{array}{l}\text { BCNU wafer } \\
\text { implantation }\end{array}$} & no & 1 & & & 1 & & \\
\hline & yes & 0.48 & $0.19-1.17$ & 0.11 & 1.12 & $0.37-3.46$ & 0.84 \\
\hline \multirow{2}{*}{$\begin{array}{l}\text { BEV available after } \\
\text { surgery }\end{array}$} & yes & 1 & & & 1 & & \\
\hline & no & 3.19 & $1.27-7.98$ & 0.013 & 3.34 & $1.11-10.1$ & 0.03 * \\
\hline
\end{tabular}

BEV: bevacizumab, CI: confidence interval, KPS: Karnofsky Performance Status.

respectively $(P=0.0008$, Fig. $2 \mathrm{~B})$. Cytoreductive surgery of recurrent GBM before BEV application can consequently contribute to the prolongation of OS after first recurrence in the BEV group.

\section{Discussion}

Once patients with GBM experience recurrence, there is no established standard treatment approach. Therapeutic options for recurrent GBM have to be carefully weighed taking into consideration the tumor location, performance status, and prognostic factors. ${ }^{14)}$ Although repeated cytoreductive surgery is one of the effective therapeutic options for improving survival in patients with recurrent GBM, ${ }^{6,15)}$ it is provided for only $10 \%-30 \%$ of patients with recurrent GBM. ${ }^{16-20)}$ According to Hervey-Jumper et al., ${ }^{21)}$ predictors of improved survival after re-operation for high-grade glioma are younger $(<50$ years $)$ were preoperative better performance status (KPS score $\geq 70$ ), a longer interval between surgery for the primary tumor and that for the recurrent lesion, smaller tumor volume, and greater extent of resection. However, these factors were not significant predictors of OS post-surgery in our study; rather, decreased KPS after cytoreductive surgery was one of the strongest clinical factors affecting OS after recurrence. If done carefully, repeated cytoreductive surgery for recurrent GBM does not negatively affect the patient's clinical condition.

To date, the majority of previous studies showed that the median interval between repeated surgery and death was less than 12 months in patients with recurrent GBM who were initially treated with the Stupp protocol. ${ }^{17,22)}$ Brandes et al. ${ }^{22)}$ reported that the median survival from second surgery for recurrent GBM treated with the Stupp protocol was 11.4 months, and the survival time was influenced by the extent of resection and 06-Methylguanine-DNA methyltransferase (MGMT) methylation status. In our study, cytoreductive surgery for recurrent GBM treated with the Stupp protocol was only 8.1 months before BEV approval, although almost all patients had gross total resection. This prognosis corresponds with prognoses in previous studies, suggesting that cytoreductive surgery makes only a limited contribution to the prolongation of the prognosis and appropriate palliative chemotherapy after cytoreductive surgery should be available.

On the other hand, our study found that after BEV approval, the prognosis of patients who received cytoreductive surgery improved significantly. As in our study, several retrospective studies reported survival prolongation achieved by the combination of cytoreductive surgery and palliative chemotherapy in the patients with recurrent GBM. ${ }^{2324)}$ De Bonis et al. ${ }^{24)}$ showed that patients treated with surgery 
and adjuvant chemotherapy had a median OS after recurrence of 14 months, compared with patients treated with chemotherapy alone who had OS after recurrence of only 8 months. Azoulay et al. ${ }^{23)}$ showed that the median survival from the time of progression was 10 months in patients with repeat surgery followed by salvage chemotherapy, compared to 6.56 months in patients with salvage chemotherapy alone. Both studies demonstrated the survival benefit of combination cytoreductive surgery with salvage chemotherapy. However, salvage chemotherapies in these studies were not uniform and they included TMZ, lomustine, procarbazine, irinotecan, and others, as well as BEV. In our study, the median OS-R of patients who received cytoreductive surgery followed by BEV treatment was 16.3 months. Compared with a previous similar investigation of repeated craniotomy for recurrent GBM, survival in our series appeared to be better. One of the reasons for these better outcomes might be that cytoreductive surgery could delay the application of BEV when gross total resection of a recurrent lesion was achieved. In this series, 10 of 18 patients (56\%) who received cytoreductive surgery in the BEV group did not use BEV immediately after surgery. There are previous retrospective studies suggesting that the delay of BEV therapy may not be associated with diminished efficacy. ${ }^{25,26)}$ Piccioni et al. demonstrated that there were no differences in survival after BEV application when stratified by the recurrence in which patients grouped into first, second, or third or more recurrences. They argued that the patients have "fixed" survival from when BEV is initiated, and thus delayed use of BEV may be preferable. ${ }^{25)}$ We suggest effective cytoreductive surgery can defer starting BEV application, leading to prolongation of OS after the first recurrence.

To the best of our knowledge, there are few studies that have focused on the impact that cytoreductive surgery has on the prognosis for recurrent glioma prior to BEV. Clark et al. ${ }^{27)}$ compared the survival of patients with recurrent GBM who received repeated craniotomy during BEV treatment with patients who received repeated craniotomy without BEV treatment in a UCSF study. Since patients who received BEV preoperatively had a worse postoperative OS and a higher perioperative morbidity rate than patients not receiving preoperative $\mathrm{BEV}$, they concluded that the indication of repeat surgery in patients in whom $\mathrm{BEV}$ treatment failed must be carefully weighed against the intended benefit and risk of surgery. Considering their conclusions together with our study, BEV should be considered as an actual lastline chemotherapy for patients with recurrent GBM. ${ }^{28,29)}$
Our study is limited by its retrospective nature, the inclusion of only a small number of patients from a single institution, and the lack of data on the molecular status of the tumors, especially MGMT methylation status. Although the prognostic values of MGMT methylation status in recurrent GBMs may not be as strong as primary GBMs, ${ }^{26)}$ their imbalanced distribution may affect survival. In addition, the difference in OS-R within the treatment era could be potentially influenced by evolving surgical techniques and support devices. To obtain more reliable results, further accumulation of clinical cases from multiple institutions is warranted.

In conclusion, as compared to the survival contribution of surgery in the pre-BEV era, cytoreductive surgery prior to BEV for recurrent GBM might contribute to survival prolongation. If effective resection without neurologic deterioration is feasible, patients with recurrent GBM should be considered for maximum cytoreduction before treatment with BEV.

\section{Acknowledgments}

The authors would like to thank Enago (www.enago. jp) for the English language review.

\section{Conflicts of Interest Disclosure}

The authors declare that they have no conflict of interest.

\section{References}

1) Stupp R, Hegi ME, Mason WP, et al.: Effects of radiotherapy with concomitant and adjuvant temozolomide versus radiotherapy alone on survival in glioblastoma in a randomised phase III study: 5-year analysis of the EORTC-NCIC trial. Lancet Oncol 10: 459-466, 2009

2) Erdem-Eraslan L, van den Bent MJ, Hoogstrate Y, et al.: Identification of patients with recurrent glioblastoma who may benefit from combined bevacizumab and CCNU therapy: a report from the BELOB trial. Cancer Res 76: 525-534, 2016

3) Taal W, Oosterkamp HM, Walenkamp AM, et al.: Single-agent bevacizumab or lomustine versus a combination of bevacizumab plus lomustine in patients with recurrent glioblastoma (BELOB trial): a randomised controlled phase 2 trial. Lancet Oncol 15: 943-953, 2014

4) Wick W, Gorlia T, Bendszus M, et al.: Lomustine and bevacizumab in progressive glioblastoma. $N$ Engl J Med 377: 1954-1963, 2017

5) Friedman HS, Prados MD, Wen PY, et al.: Bevacizumab alone and in combination with irinotecan in recurrent glioblastoma. J Clin Oncol 27: 4733-4740, 2009 
6) Bloch O, Han SJ, Cha S, et al.: Impact of extent of resection for recurrent glioblastoma on overall survival: clinical article. J Neurosurg 117: 1032-1038, 2012

7) Perrini P, Gambacciani C, Weiss A, et al.: Survival outcomes following repeat surgery for recurrent glioblastoma: a single-center retrospective analysis. J Neurooncol 131: 585-591, 2017

8) Ringel F, Pape H, Sabel M, et al.: Clinical benefit from resection of recurrent glioblastomas: results of a multicenter study including 503 patients with recurrent glioblastomas undergoing surgical resection. Neuro Oncol 18: 96-104, 2016

9) Sastry RA, Shankar GM, Gerstner ER, Curry WT: The impact of surgery on survival after progression of glioblastoma: a retrospective cohort analysis of a contemporary patient population. J Clin Neurosci 53: 41-47, 2018

10) Ortega A, Sarmiento JM, Ly D, et al.: Multiple resections and survival of recurrent glioblastoma patients in the temozolomide era. J Clin Neurosci 24: 105-111, 2016

11) Stupp R, Mason WP, van den Bent MJ, et al.: Radiotherapy plus concomitant and adjuvant temozolomide for glioblastoma. N Engl J Med 352: 987-996, 2005

12) Okamoto S, Shiga T, Hattori N, et al.: Semiquantitative analysis of C-11 methionine PET may distinguish brain tumor recurrence from radiation necrosis even in small lesions. Ann Nucl Med 25: 213-220, 2011

13) Ishi Y, Terasaka S, Yamaguchi S, et al.: Reliability of the size evaluation method for meningiomas: maximum diameter, ABC/2 formula, and planimetry method. World Neurosurg 94: 80-88, 2016

14) Tosoni A, Franceschi E, Poggi R, Brandes AA: Relapsed glioblastoma: treatment strategies for initial and subsequent recurrences. Curr Treat Options Oncol 17: 49, 2016

15) Quick J, Gessler F, Dützmann S, et al.: Benefit of tumor resection for recurrent glioblastoma. J Neurooncol 117: 365-372, 2014

16) Barbagallo GM, Jenkinson MD, Brodbelt AR: 'Recurrent' glioblastoma multiforme, when should we reoperate? Br J Neurosurg 22: 452-455, 2008

17) Montemurro N, Perrini P, Blanco MO, Vannozzi R: Second surgery for recurrent glioblastoma: a concise overview of the current literature. Clin Neurol Neurosurg 142: 60-64, 2016

18) Park CK, Kim JH, Nam DH, et al.: A practical scoring system to determine whether to proceed with surgical resection in recurrent glioblastoma. Neuro Oncol 15: 1096-1101, 2013
19) Park JK, Hodges T, Arko L, et al.: Scale to predict survival after surgery for recurrent glioblastoma multiforme. J Clin Oncol 28: 3838-3843, 2010

20) Ryken TC, Kalkanis SN, Buatti JM, Olson JJ, Committee ACJG: The role of cytoreductive surgery in the management of progressive glioblastoma : a systematic review and evidence-based clinical practice guideline. J Neurooncol 118: 479-488, 2014

21) Hervey-Jumper SL, Berger MS: Reoperation for recurrent high-grade glioma: a current perspective of the literature. Neurosurgery 75: 491-499; discussion 498499, 2014

22) Brandes AA, Bartolotti M, Tosoni A, et al.: Patient outcomes following second surgery for recurrent glioblastoma. Future Oncol 12: 1039-1044, 2016

23) Azoulay M, Santos F, Shenouda G, et al.: Benefit of re-operation and salvage therapies for recurrent glioblastoma multiforme: results from a single institution. J Neurooncol 132: 419-426, 2017

24) De Bonis P, Fiorentino A, Anile C, et al.: The impact of repeated surgery and adjuvant therapy on survival for patients with recurrent glioblastoma. Clin Neurol Neurosurg 115: 883-886, 2013

25) Piccioni DE, Selfridge J, Mody RR, et al.: Deferred use of bevacizumab for recurrent glioblastoma is not associated with diminished efficacy. Neuro Oncol 16: 815-822, 2014

26) Schaub C, Schäfer N, Mack F, et al.: The earlier the better? Bevacizumab in the treatment of recurrent MGMT-non-methylated glioblastoma. J Cancer Res Clin Oncol 142: 1825-1829, 2016

27) Clark AJ, Lamborn KR, Butowski NA, et al.: Neurosurgical management and prognosis of patients with glioblastoma that progresses during bevacizumab treatment. Neurosurgery 70: 361-370, 2012

28) Wenger KJ, Wagner M, You SJ, et al.: Bevacizumab as a last-line treatment for glioblastoma following failure of radiotherapy, temozolomide and lomustine. Oncol Lett 14: 1141-1146, 2017

29) Heiland DH, Masalha W, Franco P, Machein MR, Weyerbrock A: Progression-free and overall survival in patients with recurrent Glioblastoma multiforme treated with last-line bevacizumab versus bevacizumab/lomustine. J Neurooncol 126: 567-575, 2016

\footnotetext{
Corresponding author: Shigeru Yamaguchi, MD Department of Neurosurgery, Faculty of Medicine, Hokkaido University, North 15 West 7, Kita-ku, Sapporo Hokkaido 060-8638, Japan. e-mail: yama-shu@med.hokudai.ac.jp
} 